\title{
Utility of a standardized training program for endoscopic diagnosis of early gastrointestinal neoplasia
}

\section{다 (1) $\odot$}

\section{Authors}

Philip W. Chiu, , , Yasushi Sano ${ }^{2, ~}$, Noriya Uedo ${ }^{3,}{ }^{*}$, Rajvinder Singh ${ }^{4}$, Enders K.W. Ng ${ }^{1}$, Tiing Leong Aang ${ }^{5}$, Han Mo Chiu ${ }^{6}$, Shiaw-Hooi Ho ${ }^{7}$, Rupa Banerjee ${ }^{8}$, Shinji Tanaka ${ }^{9}$, Xiao Bo Li ${ }^{10}$, Fang Yao ${ }^{11}$, James Y.W. Lau ${ }^{11}$, Kenshi Yao $^{12}$

Institutions

1 Department of Surgery, Institute of Digestive Disease, The Chinese University of Hong Kong, Hong Kong S.A.R., China

2 Gastrointestinal Center \& iMEC (Institute of Minimallyinvasive Endoscopic Care), Sano Hospital, Kobe, Japan

3 Department of Gastrointestinal Oncology, Osaka Medical Center for Cancer and Cardiovascular Diseases, Osaka, Japan

4 Department of Gastroenterology, Lyell McEwin \& Modbury Hospitals, University of Adelaide, Australia

5 Department of Gastroenterology \& Hepatology, Changi General Hospital, Singapore

6 Division of Gastroenterology, Department of Internal Medicine, National Taiwan University, Taiwan

7 Department of Gastroenterology, University of Malaya, Malaysia

8 Department of Medical Gastroenterology, Asian Institute of Gastroenterology, Hyderabad, India

9 Department of Endoscopy, Hiroshima University Hospital, Hiroshima, Japan

10 Department of Gastroenterology, Renji Hospital, Shanghai, China

11 Department of Gastroenterology, Peking Union Medical College Hospital, Beijing, China

12 Department of Gastroenterology, Fukuoka University Chikushi Hospital, Fukuoka, Japan

submitted 22.3.2018

accepted after revision 18.9.2018

Bibliography

DOI https://doi.org/10.1055/a-0854-3525 |

Endoscopy International Open 2019; 07: E452-E458

(C) Georg Thieme Verlag KG Stuttgart · New York

ISSN 2364-3722

Corresponding author

Prof. Philip WY Chiu, Department of Surgery, Faculty of Medicine, The Chinese University of Hong Kong, 4th floor,
Lui Che Woo Clinical Science Building, Prince of Wales

Hospital, 30-32 Ngan Shing Street, Shatin,

Hong Kong S.A.R., China

Fax: +85226377974

philipchiu@surgery.cuhk.edu.hk

\section{ABSTRACT}

Background and study aims Image enhanced endoscopy (IEE) allows endoscopists to improve recognition and characterization of gastrointestinal neoplasia. The Asian Novel Bio-Imaging and Intervention Group (ANBIG) conducted a standardized training program in endoscopic diagnosis and treatment of early gastrointestinal cancers in Asia. We embarked on a study to investigate the effect of this module on endoscopic diagnosis of early gastrointestinal neoplasia.

Methods This prospectively collected database was from workshops conducted on training for endoscopic diagnosis of early gastrointestinal neoplasia. All workshops were conducted in a standardized format, which included a pretest, a learning phase consisting of didactic lectures, case discussion, and live demonstration followed by a post-test to assess knowledge gained. The pretest and post-training tests were standardized questions addressing four domains, including basic knowledge of imaging and diagnosis of esophageal, gastric, and colonic neoplasia.

Results From November 2013 to November 2016, 41 ANBIG workshops were conducted in 13 countries. A total of 1863 delegates and 40 faculty participated in these workshops. Of the delegates, 627 completed both tests. There was a significant improvement after training in all domains of the tests. There was a trend in general lack of knowledge across all domains for delegates from "low" healthcare cost countries before training. All delegates demonstrated significant improvement in knowledge of all domains after the workshop irrespective of whether they were from "high" or "low" healthcare cost per capita countries.

Conclusion A standardized teaching program on IEE improved the diagnostic ability and quality of endoscopists in recognizing early gastrointestinal neoplasia in Asia.

\footnotetext{
* These authors contributed equally.
} 


\section{Introduction}

Cancers of the gastrointestinal tract represent the most common cancers worldwide, accounting for significant number of deaths annually. In 2012, gastric cancer was the third leading cause of cancer death while colorectal cancer was the third commonest cancer worldwide [1]. The prognosis for any cancer depends on stage of the disease at diagnosis. For instance, 5year survival for early intramucosal gastrointestinal cancers is generally more than $80 \%$ [2]. As with most cancers, the neoplastic process of gastrointestinal cancers has its beginnings from genetic alternations of mucosal cells. These early cancers are hence amenable to endoscopic treatment [3]. Numerous studies have been performed confirming the efficacy of novel endoscopic technologies in recognition and characterization of early gastrointestinal neoplasia $[3,4]$. However, the proportion of early stage gastrointestinal cancers being diagnosed differs among various countries. In Japan and Korea, more than $70 \%$ of patients with gastric cancers were diagnosed at an early stage resulting in excellent 5 -year survival rates $[5,6]$. Suzuki, et al reviewed 1226 patients with early gastric cancer at the $\mathrm{Na}$ tional Cancer Center Hospital, Tokyo, and found that most of these early gastric cancers were detected by endoscopy outside of mass screening programs (which were performed using photofluorography) [7]. Performing a good-quality examination is hence paramount to improve outcomes of gastrointestinal cancers. One of the key initiatives that may improve diagnostic yield of endoscopy for early gastrointestinal cancers is education and training. Given this background, a professional group consisting of experts in endoscopic diagnosis (the Asian Novel Bio-Imaging and Intervention Group; ANBIG) was established in 2013. The main objective of the group is to improve endoscopic diagnosis and treatment of early gastrointestinal neoplasia through a standardized educational program in Asia. This was a prospective study to investigate the effect of this program on improvement in endoscopic diagnosis of early gastrointestinal neoplasia.

\section{Methods}

A prospective cohort study was performed to investigate the effect of a standardized training program for endoscopic diagnosis and management of early gastrointestinal neoplasia in 13 Asian countries. Members of ANBIG were divided into upper and lower gastrointestinal groups and all training materials were standardized, including standard slide sets for education on recognition and classification of early esophageal, gastric and colorectal neoplasia.

\section{Standardized training program}

The objective of the standardized training program was to systematically conduct teaching on endoscopic diagnosis and treatment of early gastrointestinal cancers to enhance learning and knowledge acquirement. The format of the program was designed as follows. A pre-training test was initially conducted through a wireless polling system. The test consisted of 29 multiple-choice questions dedicated to testing of knowledge in four domains, including basic knowledge of image enhanced endoscopy, diagnosis of early esophageal, gastric and colorectal neoplasia ( Fig.1). There were four questions on basic knowledge; eight questions on diagnosis of early esophageal neoplasia; eight questions on diagnosis of early gastric cancer and nine questions on diagnosis of early colorectal neoplasia. A structured training program was then delivered to participants. This format included didactic 30-minute lectures on each topic, illustration of cases as well as live demonstration or video presentation. Some of the typical teaching lectures can be found at http://www.anbig.org. These workshops also included hands-on training on endoscopic mucosal resection (EMR) and endoscopic submucosal dissection (ESD).

The general approach to endoscopic diagnosis of early esophageal, gastric, and colorectal neoplasia was also standardized into a two-step approach. The first step was recognition of abnormal changes during diagnostic endoscopy under white light endoscopy and narrow-band imaging (NBI). The second step was characterization of abnormal changes during endoscopy ( $\triangleright$ Fig. 1 and $>$ Fig.2). Characterization was based on changes in microsurface and microvascular architecture. Characterization of early gastric cancer was based on MS and MV classification reported by Yao, et al [8]. Characterization of early colorectal neoplasia was based on NICE classification reported by Sano, et al [9], while characterization of early esophageal neoplasia was based on IPCL pattern classification reported by Inoue, et al [10]. After training, all delegates completed the post-training tests with same sets of questions. Results of pretests and post-tests were then compared and reviewed. All delegates received feedback on the final answers, discussing the salient features of each question in a structured and methodical manner at the end of the workshop.

\section{Data collection}

Data from the pretests and post-tests that were prospectively collected through the wireless polling system were entered into a database. Delegates who had not completed either pretests or post-tests were excluded from the study. Because AN$\mathrm{BIG}$ workshops were conducted in various cities in various countries across Asia, we anticipated a difference in healthcare development. We compared results of pretests and post-tests among ANBIG workshops conducted in countries with "high" and "low" healthcare cost per capita according to information obtained from the World Health Organization and Organization for Economic Cooperation and Development (OECD) library $[11,12]$. We defined >USD\$1000 per capita as "high" healthcare cost countries, and <USD $\$ 1000$ as "low" healthcare cost countries. Given these definitions, Australia, Hong Kong, Singapore, South Korea, and Taiwan were considered high healthcare cost per capita countries, while China, India, Indonesia, Malaysia, Mongolia, Myanmar, Philippines, Sri Lanka, and Thailand were classified as low healthcare cost per capita countries. 


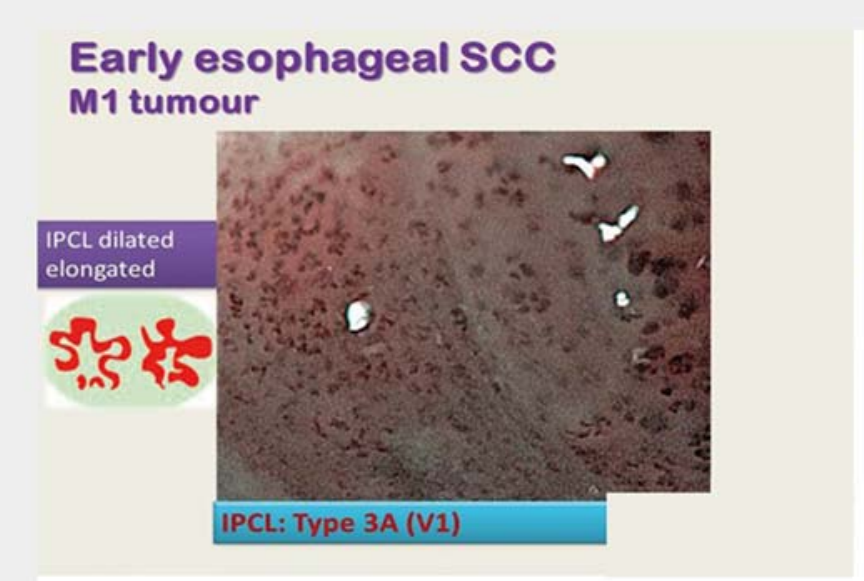

\section{Early Esophageal SCC Submucosal ( $\geq$ SM2)}

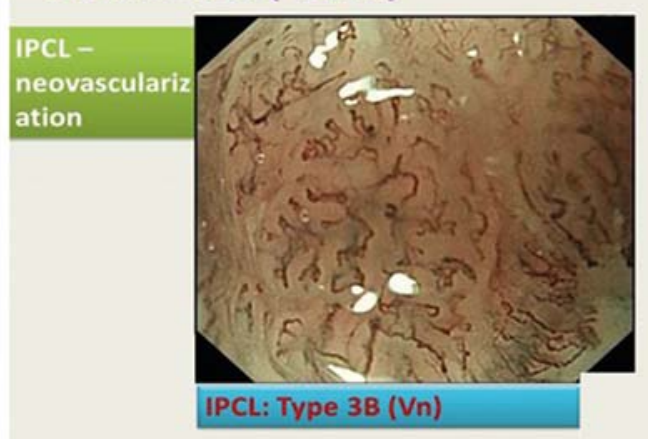

\section{Magnifying NBI images of $\mathbf{H}$. pylori negative corpus mucosa}
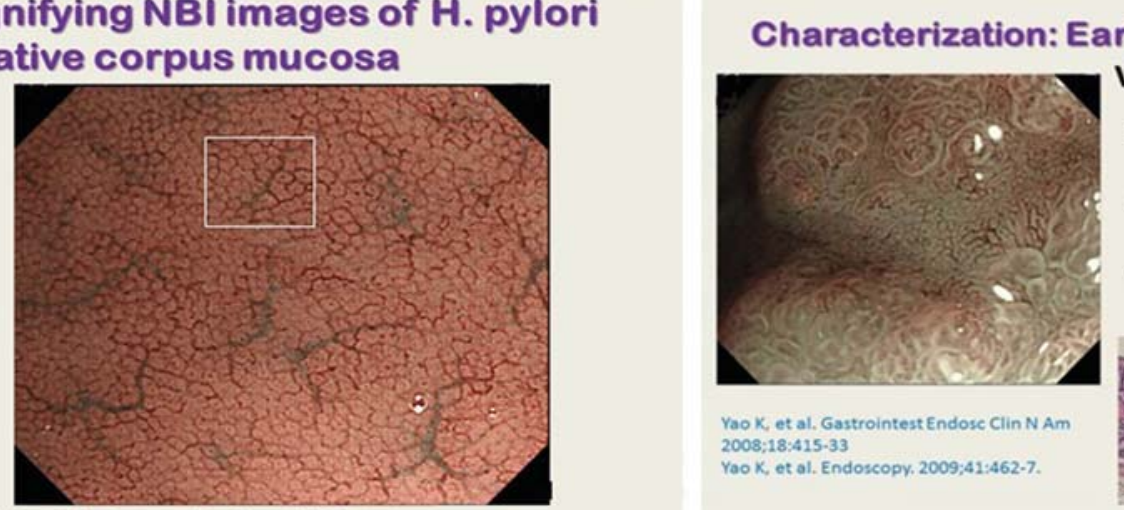

Yao K, et al. Gastrointest Endose Clin N Am 2008:18:415-33

Yoo K, et al. Endoscopy. 2009;41:462-7.

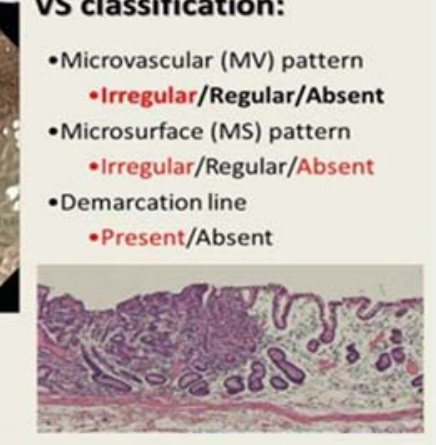

Osaka Medical Center for Cancer and Cardiovascular Diseases

Fig. 1 Standard training slides on recognition and characterization of early gastric and esophageal neoplasia.

\section{Statistical analysis}

Categorical data were compared using the Chi-square test and continuous data were compared with the Student's $t$-test. Results were considered statistically significant if the $P$ value was less than 0.05 .

\section{Results}

From November 2013 to March 2016, a total of 41 ANBIG workshops were conducted in 13 different cities and countries in Asia. A total of 1863 delegates and 40 faculties participated in these workshops, and data from 627 of the delegates who completed both pretests and post-tests were analyzed. Forty faculty members/experts (local and regional) participated in conducting the training courses. When comparing the overall results, there was significant improvement in the post-test results when compared to pretest results across all the domains $(P<0.0001)$ ( Fig. 3$)$.

The workshops were divided into those conducted at high and low healthcare cost per capita countries. Comparing the accuracy of pretests and post-tests, there was a lower accuracy in pretest knowledge before training for participants in workshops conducted in low healthcare cost per capita countries compared to those conducted in high healthcare cost per capi- ta countries ( $>$ Fig. 4 ). There was a trend towards a lower level of knowledge across all domains before training for delegates who participated in courses organized in low healthcare cost countries, while all delegates demonstrated significant improvement in knowledge of all domains after the training to similar levels, irrespective for whether they were from high or low healthcare cost per capita countries.

\section{Discussion}

The prognosis for gastrointestinal cancers is closely related to stage of the disease [2]. Compared to advanced gastrointestinal cancers, early stage disease carries an excellent prognosis with 5 -year survival of more than $80 \%[13,14]$. The prognosis for early gastrointestinal cancers is excellent as the risk of lymph node metastasis is minimal [15]. Endoscopic en-bloc resection with ESD achieves adequate resection margins and significantly reduced risk of local recurrence [16-18]. Compared to surgery, endoscopic resection achieves similar oncological outcomes with better recovery. However, diagnosis of gastrointestinal cancers at early stage imposes major difficulties in most countries worldwide. One of the major issues is that early gastrointestinal cancers are often characterized by subtle changes of the microstructure and microvascular patterns of 


\section{'national Colorectal Endoscopic (NICE) Classification}

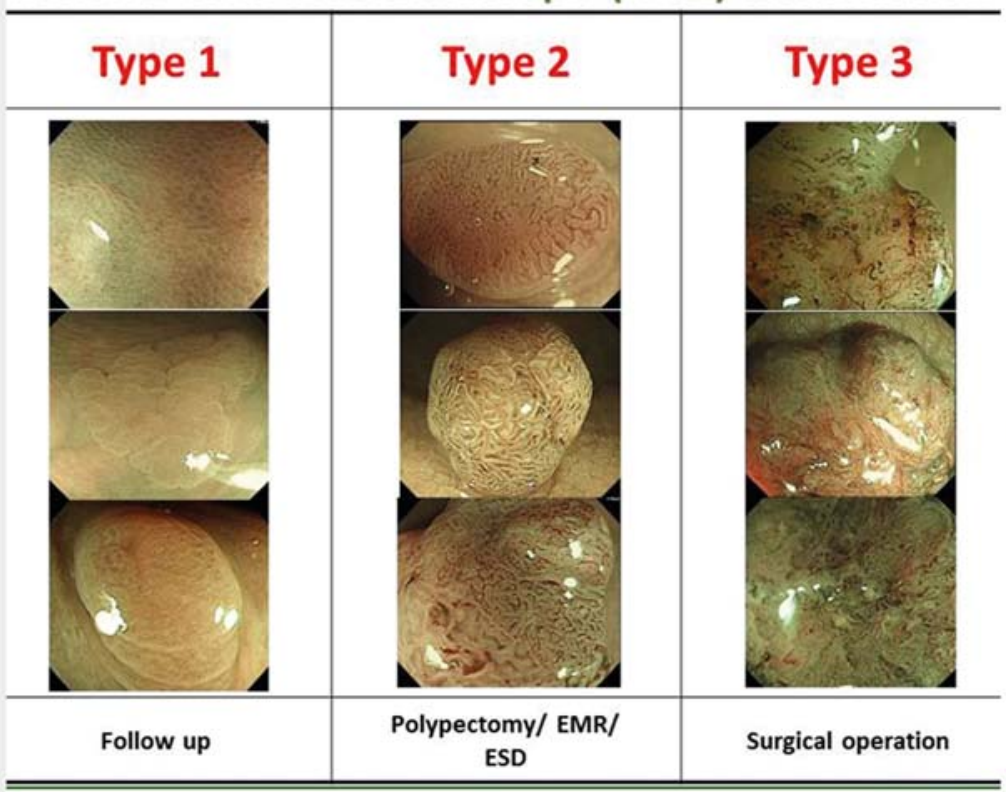

asushi SANO M.D., Ph.D.

irector and Chief of Gastrointestinal Center \& iMEC
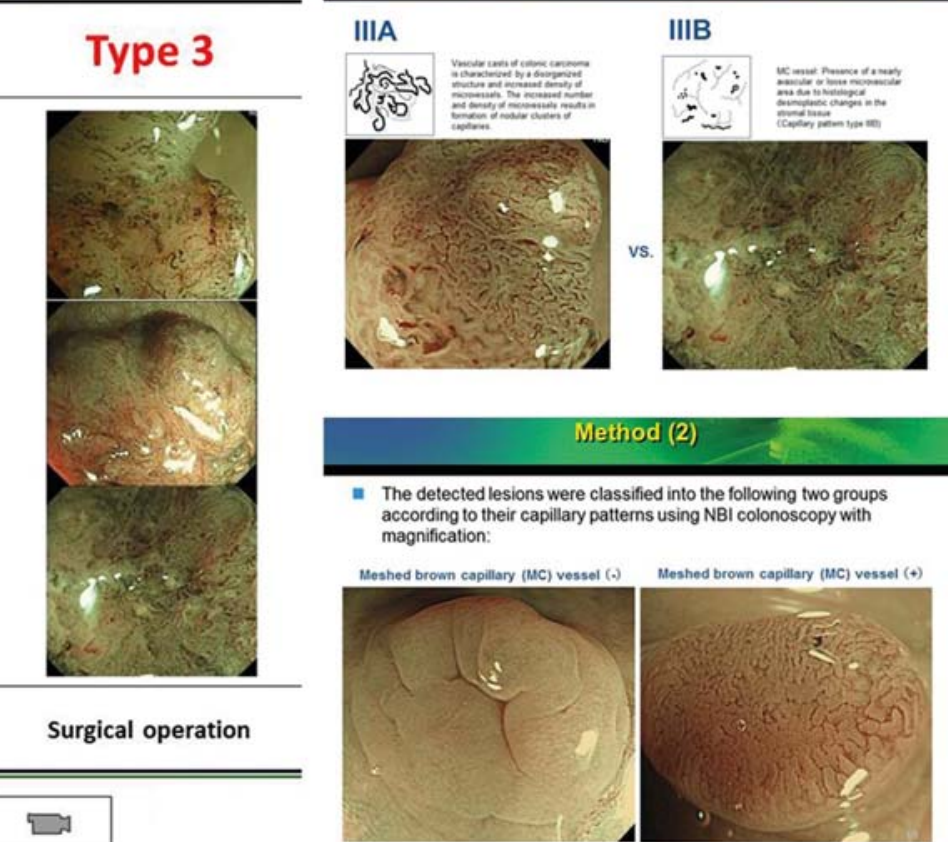

II The detected lesions were classified into the following two groups according to their capillary patterns using NBI colonoscopy with magnification:

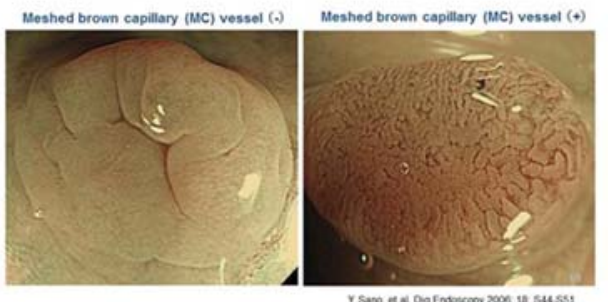

- Fig. 2 Standard training slides on recognition, characterization and classification of early colorectal neoplasia.

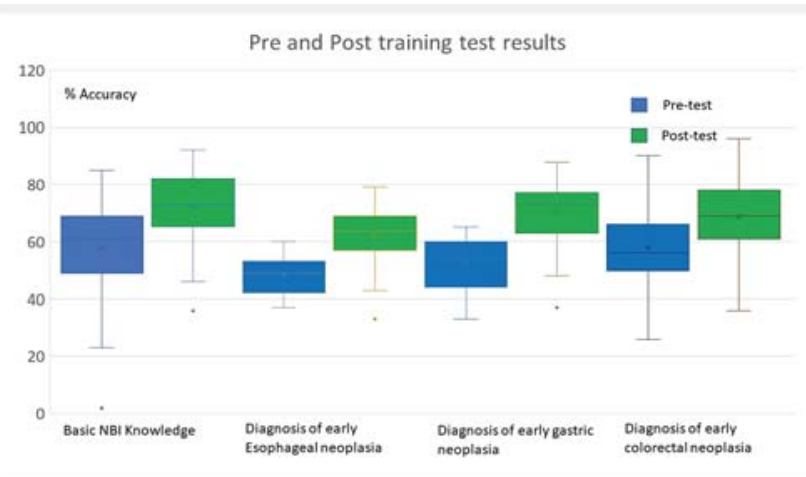

Fig. 3 Comparison of pretest and post-test results from delegates who participated in the training courses on endoscopic diagnosis of early gastrointestinal cancers.

the mucosa. Macroscopic changes in early gastrointestinal cancers may only be represented by minute architectural and color changes. The key to recognition of these subtle changes in endoscopy depends on allowing reasonable time for careful endoscopic examination as well as knowledge and experience to recognize these changes.

There is limited research on the effect of a structured training program on improving endoscopic diagnosis of early gastrointestinal cancers. Dias-Silva et al. investigated the effect of a systematic learning program using Web-based videos on NBI for diagnosis of precancerous gastric lesions with six gastroenterologists [19]. The learning system included initial presenta- tion of classification for precancerous gastric lesions followed by 20 tests each containing 10 randomly selected NBI videos for gastric mucosa. The participants were asked to diagnosis these lesions with respect to: 1 . Standard diagnostic pattern; 2. Presence or absence of light blue crest sign; and 3. Presence or absence of Helicobacter pylori infection. There was a significant improvement in sensitivity and specificity for diagnosis of intestinal metaplasia as well as dysplasia after training with increasing number of videos. Yao et al. conducted a large prospective, multicenter, randomized controlled trial to assess efficacy of an e-learning system for endoscopic diagnosis of early gastric cancer including endoscopists from 35 countries around the world [20]. The program was built on an electronic platform, and the participants first performed a pretest with 40 sets of endoscopic images of cancerous and non-cancerous lesions. They were then educated by e-learning platform with lectures on video clips and slides on the technique and knowledge for diagnosis of early gastric cancers. They then received training on detection and differentiation of early gastric cancer on 100 cases. A post-test was conducted after completion of training. Compared to the pretest, there was significant improvement in the post-test mean score for participants who received e-learning as compared to those who did not. The advantage of Internet-based lectures is the opportunity for standardization and delivery of learning activity to medical practitioners all over the world easily and cost-effectively. Our training activity was based on a conventional classroom-style module. This allowed a more personal approach. Lectures were intertwined with an interactive question, answer, and discussion format. 


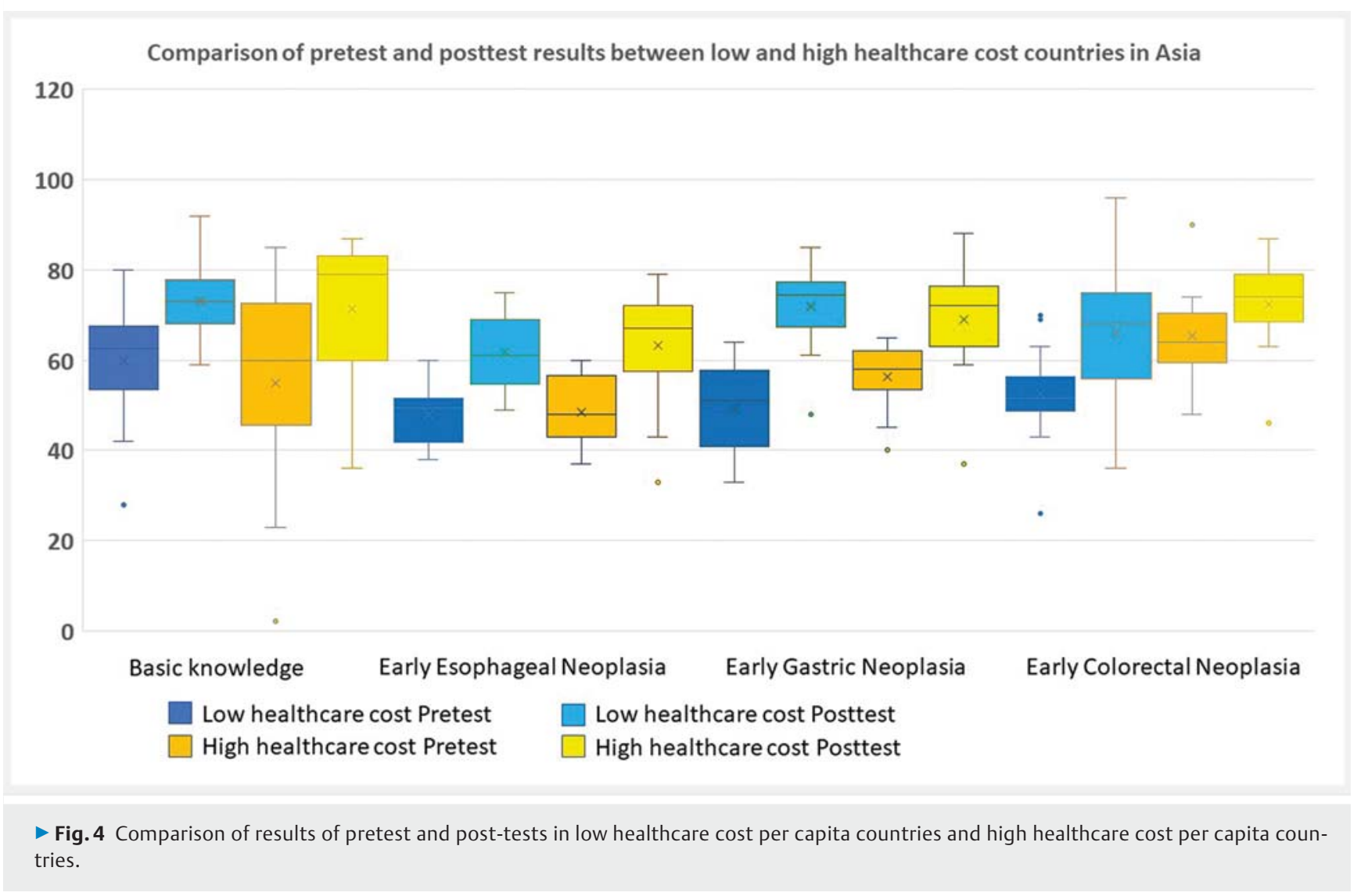

Participants were encouraged to interact with the experts. We also performed live demonstrations and hands-on training to further elucidate the importance of endoscopic imaging and how it may influence clinical practice.

One of the main difficulties in conducting an educational program for endoscopic diagnosis of early gastrointestinal cancers is changing the mindset of trained endoscopists. The ANBIG program was designed to specifically alter this. Traditionally, endoscopy is performed to explain a patients' symptoms or to investigate an abnormal finding from another diagnostic examination [21]. Most early gastric cancers are diagnosed in patients who have received upper gastrointestinal endoscopy for other reasons. Community-based case control studies of endoscopic screening in Japan have demonstrated significant reduction in gastric cancer mortality in patients who participated in an endoscopic screening program compared to those who were never screened $[22,23]$. There is, however, a wide range of sensitivity in detection of early gastric cancer during endoscopic screening $[24,25]$. Gotoda et al. pointed out that currently, there is no universal standard for performance or number of images recorded during esaphogastroduodenoscopy [21]. Yao et al. proposed the systematic screening protocol for the stomach (SSS) to ensure a complete observation is made while performing endoscopy [20]. This concept was introduced to participants in the workshop.

There are also many studies evaluating the learning curve for NBI-based diagnosis using training modules for differentiating neoplastic and non-neoplastic lesions in the colon. Some stud- ies reported that high accuracy can be achieved ex vivo by nonexperts after a short and simple training course or a computerized training program [25-28]. Higashi et al. reported that an intensive, 1-hour, interactive training program allowed improvement in accuracy from $53 \%$ to $66 \%$ by four non-endoscopists, and from $65 \%$ to $88 \%$ by four endoscopists without $\mathrm{NBI}$ experience using still images [56]. Rastogi et al. showed a significant improvement in accuracy (from $64 \%$ to $81 \%$ ) and proportion of high-confidence diagnosis (from $49 \%$ to $69 \%$ ) on a set of $40 \mathrm{NBI}$ videos after training with a 20 -minute computerbased module with video clips [28]. Other studies, however, have demonstrated no statistically significant improvement in in vivo accuracy after video-based instruction [29,30]. Ladabaum and colleagues reported that 12 of 13 endoscopists were able to identify adenomas with greater than $90 \%$ accuracy in tests using two different sets of 25 endoscopic NBI still images at the end of computer-based training. However, disappointingly, only three of 12 endoscopists achieved accuracy of greater than $90 \%$ [29]. Assessment during colonoscopy is different from evaluation of still endoscopic images as the "realtime" factor is negated with the latter. Diagnosing colorectal polyps in vivo requires endoscopists to take clear crisp images, which can sometimes be difficult in a spasmodic or debris-filled colon. The ANBIG program differed from the above as this was an "onsite" training program.

There are several limitations to this study. Firstly, outcome assessment was based on accuracy in recognizing and classifying early gastrointestinal cancers. That assessment may not be 
as robust as detection rate for early gastrointestinal cancers or adenoma detection rate before and after the structured training program. Collection of outcomes on diagnostic endoscopy from individual participants will be difficult, as that involves more complex data collection and handling. Second, the ANBIG program was designed as a one-time training on diagnosis of early gastrointestinal cancers. Although we have developed an educational website to enhance continuous training, continuity of improvement in endoscopic diagnosis depends on the enthusiasm of participating endoscopists. This program was not designed to assess long-term durability. Plans are underway to look into this, including conducting of a cohort study to investigate the effect on detection of early gastrointestinal neoplasia before and after training. Dai and colleagues investigated diagnostic accuracy during colonoscopy using three sets of 15 lesions after a 4-hour training program. In the first set of 15 lesions, diagnostic accuracy for both target and non-target lesions was not satisfied. In the second set and after providing feedback (including histopathological results), less experienced endoscopists improved their diagnostic accuracy for non-target lesions (from $70.0 \%$ to $96.7 \%$ ) and target lesions (from $78.3 \%$ to $96.7 \%$ ) [31]. Continuous feedback may be an important factor in achieving improvement in accuracy in real clinical practice. Feedback was provided after the post-test in our study but only during discussion of the test results.

Detection and characterization of early gastrointestinal neoplasia remain two distinctly important entities.

The unmet need for a structured educational and teaching program was the catalyst that led to formation of ANBIG. The program was performed onsite across a vast spectrum of conditions in many different countries to bridge a recognized knowledge gap. Overall, feedback from the participants was positive, especially on how valuable the various classification systems for early gastrointestinal cancers were to them.

\section{Conclusion}

In conclusion, a standardized training program on IEE improved the diagnostic ability of endoscopists in recognizing early gastrointestinal neoplasia in Asia. The ANBIG program is easily grasped and reproducible. The authors believe that there is huge potential for this to be introduced to other continents according to regional needs.

\section{Acknowledgement}

The Asian Novel Imaging and Intervention Group (ANBIG) is a non-government organization established by experts in the field of gastrointestinal endoscopy with the aim to improve endoscopic diagnosis and treatment of early gastrointestinal cancers through education and training. ANBIG receives sponsorship from healthcare industries, including Olympus Co Ltd and Takeda Co Ltd, and organizes education and training courses under an independent steering committee.
Competing interests

The Asian Novel Imaging and Intervention Group (ANBIG) is a non-government organization established by experts in the field of gastrointestinal endoscopy with the aim to improve endoscopic diagnosis and treatment of early gastrointestinal cancers through education and training. ANBIG receives sponsorship from healthcare industries, including Olympus Co Ltd and Takeda Co Ltd, and organizes education and training courses under an independent steering committee.

\section{References}

[1] Torre LA, Bray F, Siegel RL et al. Global cancer statistics, 2012. CA Cancer J Clin 2015; 65: 87-108

[2] Everett SM, Axon AT. Early gastric cancer in Europe. Gut 1997; 41: $142-150$

[3] Veitch AM, Uedo N, Yao K et al. Optimizing early upper gastrointestinal cancer detection at endoscopy. Nat Rev Gastroenterol Hepatol 2015; 12: $660-667$

[4] Ezoe $\mathrm{Y}$, Muto $\mathrm{M}$, Uedo $\mathrm{N}$ et al. Magnifying narrowband imaging is more accurate than conventional white-light imaging in diagnosis of gastric mucosal cancer. Gastroenterology 2011; 141: 2017-2025

[5] Lim D, Ha M, Song I. Trends in major cancer mortality in Korea, 19832012, with a joinpoint analysis. Cancer Epidemiol 2015; 39: 939-946

[6] Hamashima C, Ogoshi K, Narisawa R et al. Impact of endoscopic screening on mortality reduction from gastric cancer. World J Gastroenterol 2015; 21: $2460-2466$

[7] Suzuki H, Gotoda T, Sasako M et al. Detection of early gastric cancer: misunderstanding the role of mass screening. Gastric Cancer 2006; 9: 315-319

[8] Yao K, Anagnostopoulos GK, Ragunath K. Magnifying endoscopy for diagnosing and delineating early gastric cancer. Endoscopy 2009; 41 : $462-467$

[9] Hewett DG, Kaltenbach T, Sano Y et al. Validation of a simple classification system for endoscopic diagnosis of small colorectal polyps using narrow band imaging. Gastroenterology 2012; 143: 599-607

[10] Inoue H, Honda T, Yoshida T et al. Ultra-high magnification endoscopy of normal esophageal mucosa. Digestive Endoscopy 1996; 8: $134-138$

[11] World Health Organization. Health financing. New perspectives on global health spending for universal health coverage. Available at: https://www.who.int/health_financing/topics/resource-tracking/ new-perspectives/en/ [Accessed January 28, 2019]

[12] OECD iLibrary. Health expenditure per capita - Health at a Glance . 2013: Available at: http://www.oecd-ilibrary.org/social-issues-migration-health/health-at-a-glance-2013/health-expenditure-per-capita_health_glance-2013-64-en [Accessed January 28, 2019]

[13] Chapelle N, Bouvier AM, Manfredi S et al. Early gastric cancer: trends in incidence, management, and survival in a well-defined French population. Ann Surg Oncol 2016; 23: 3677-3683

[14] Torre LA, Siegel RL, Ward EM et al. global cancer incidence and mortality rates and trends-an update. Cancer Epidemiol Biomarkers Prev 2016; 25: $16-27$

[15] Gotoda T, Yanagisawa A, Sasako M et al. Incidence of lymph node metastasis from early gastric cancer: estimation with a large number of cases at two large centers. Gastric Cancer 2000; 3: 219-225

[16] Tanabe S, Ishido K, Matsumoto T et al. Long-term outcomes of endoscopic submucosal dissection for early gastric cancer: a multicenter collaborative study. Gastric Cancer 2017; 20: 45-52 
[17] Saito Y, Uraoka T, Matsuda T et al. Endoscopic treatment of large superficial colorectal tumors: a case series of 200 endoscopic submucosal dissections (with video). Gastrointest Endosc 2007; 66: 966 973

[18] Tsujii Y, Nishida T, Nishiyama O et al. Clinical outcomes of endoscopic submucosal dissection for superficial esophageal neoplasms: a multicenter retrospective cohort study. Endoscopy 2015; 47: 775 - 783

[19] Dias-Silva D, Pimentel-Nunes P, Magalhaes J et al. The learning curve for narrow-band imaging in the diagnosis of precancerous gastric lesions by web-based video. Gastrointest Endosc 2014; 79: 910 - 920

[20] Yao K, Uedo N, Muto M et al. Development of an E-learning system for the endoscopic diagnosis of early gastric cancer: an international multicenter randomized controlled trial. EBioMedicine 2016; 9: $140-147$

[21] Gotoda T, Uedo N, Yoshinaga S et al. Basic principles and practice of gastric cancer screening using high-definition white-light gastroscopy: Eyes can only see what the brain knows. Dig Endosc 2016; 28 : (Suppl. 01): 2-15

[22] Oshima A. A critical review of cancer screening programs in Japan. Int J Technol Assess Health Care 1994; 10: 346 - 358

[23] Hamashima C. Benefits and harms of endoscopic screening for gastric cancer. World J Gastroenterol 2016; 22: 6385-6392

[24] Choi KS, Jun JK, Park EC et al. Performance of different gastric cancer screening methods in Korea: a population-based study. PLoS One 2012; 7: e50041
[25] Higashi R, Uraoka T, Kato ] et al. Diagnostic accuracy of narrow-band imaging and pit pattern analysis significantly improved for less-experienced endoscopists after an expanded training program. Gastrointest Endosc 2010; 72: 127-135

[26] Raghavendra M, Hewett DG, Rex D. Differentiating adenomas from hyperplastic colorectal polyps: Narrow-band imaging can be learned in 20 minutes. Gastrointest Endosc 2010; 72: 572 - 576

[27] Ignjatovic A, Thomas-Gibson S, East JE et al. Development and validation of a training module on the use of narrow-band imaging in differentiation of small adenomas from hyperplastic colorectal polyps. Gastrointest Endosc 2011; 73: 128-133

[28] Rastogi A, Rao DS, Gupta N et al. Impact of a computer-based teaching module on characterization of diminutive colon polyps by using narrow-band imaging by non-experts in academic and community practice: a video-based study. Gastrointest Endosc 2014; 79: $390-398$

[29] Ladabaum U, Fioritto A, Mitani A et al. Real-time optical biopsy of colon polyps with narrow band imaging in community practice does not yet meet key thresholds for clinical decisions. Gastroenterology 2013; 144: $81-91$

[30] Kuiper T, Marsman WA, Jansen JM et al. Accuracy for optical diagnosis of small colorectal polyps in nonacademic settings. Clin Gastroenterol Hepatol 2012; 10: $1016-1020$

[31] Dai ], Shen YF, Sano Y et al. Evaluation of narrow-band imaging in the diagnosis of colorectal lesions: is a learning curve involved? Dig Endosc 2013; 25: $180-188$ 